\title{
Comparison of the seasonal patterns of asthma identified in general practitioner episodes, hospital admissions, and deaths
}

\author{
D M Fleming, K W Cross, R Sunderland, A M Ross
}

\begin{abstract}
Background-Seasonal variations in asthma are widely recognised. This study was undertaken to investigate the relative differences in seasonal patterns by age as they impact on episodes of care in general practice, hospital admissions, and deaths. Methods-General practice episode data from the Weekly Returns Service of the Royal College of General Practitioners, hospital admissions for asthma in England, and deaths registered as due to asthma in England and Wales over the years 1990-7 were examined. Age specific weekly rates of new episodes of asthma presenting to general practitioners, numbers of hospital admissions and deaths were analysed by the multiplicative decomposition method to separate secular from seasonal trends. The seasonal indices thereby obtained were plotted as three week moving averages.
\end{abstract}

Results-In children aged 0-4 and 5-14 years general practice episodes and admissions to hospital were strikingly congruent in timing and in magnitude, except in September when particularly high rates of admission (absolute and relative to general practice episodes) occurred. In the 15-44 age group there were marked mid summer peaks of general practice episodes and deaths but admissions to hospital were at about the annual average; in September/ October there were peaks of episodes and admissions whereas deaths peaked in November. In the 45-64 age group a peak in general practice episodes of asthma was evident in mid summer when admissions were about average and deaths were at a minimum; all three measures tended to increase gradually with the approach of winter. Finally, in those age over 65 years, general practice episodes of asthma, admissions to hospital, and deaths followed similar ' $U$ ' shaped patterns with substantial peaks in mid winter.

Conclusions-The seasonal pattern of asthma evolves with age. There are important differences in the seasonal pattern of general practice episodes, admissions to hospital, and deaths. Individual seasonal histories are important for the management of asthma. The combined analysis of these three data sets provides a new perspective on the epidemiology of asthma. (Thorax 2000;55:662-665)

Keywords: asthma; hospital admissions; asthma deaths; seasonality
Asthma is a common condition of fluctuating severity, variable age of onset, and seasonal determination. ${ }^{1}$ Extrapolation from a general practice morbidity survey of half a million people in England and Wales (September 1991 to August 1992) indicates that, in the population of 52 million, there were approximately 2.2 million with asthma accounting for five million consultations per annum. ${ }^{2}$ In 1992 there were 90814 hospital admissions in England ${ }^{3}$ and 1790 deaths in England and Wales ${ }^{4}$ attributed to asthma.

Seasonal patterns in the incidence of asthma attacks, in admissions to hospital, and in deaths from asthma are well recognised. Khot and Burn ${ }^{5}$ found increased mortality from asthma in England in the period July to September in subjects aged 5-34 years. Campbell and colleagues reported a peak of deaths in August in patients aged 5-14 years but in July in those aged 15-44 years. ${ }^{6}{ }^{7}$ Marks and Burney studied a long time series for both admissions and mortality from asthma and identified inconsistencies between the two; different patterns were noted in subjects aged less than 35 years and those in the oldest age groups, suggesting that different factors were responsible for exacerbations of asthma. ${ }^{8}$ Khot and colleagues, ${ }^{9}$ reporting on admissions to hospital of children between 1975 and 1981, noted a deep trough in August followed by a peak in autumn. Storr and Lenny drew attention to peaks in the admission of children to hospital immediately after school holidays, notably in September. ${ }^{10}$

Acute asthma attacks are one of the most common treatable medical emergencies. Patients are often caught unawares by them and, for some, the unpredictable nature of severity and onset causes considerable anxiety and restriction of life style. This report is concerned with the seasonal patterns of asthma attacks in the community and the relationship these bear to hospital admissions and to deaths. The recognition of clear seasonal patterns of asthma attacks in the community and in individuals may allow preventive strategies to be developed.

\section{Methods}

The following three sets of unlinked data in five age specific groups $(0-4,5-14,15-44,45-64$, and $65+$ years) covering the eight years $1990-7$ were used:

(1) Weekly incidence data of asthma episodes reported in the Weekly Returns Service of the Royal College of General Practitioners. ${ }^{11}{ }^{12}$ A new episode of asthma

\footnotetext{
2 May 2000

Accepted for publication

17 May 2000

Received 13 August 1999 Returned to authors 3 November 1999 3 November 1999
Revised version received
} 
in general practice (GP episode) is reported when a patient consults with an exacerbation of asthma but not when consulting for management review or solely to obtain further medication.

(2) Hospital Finished Consulting Episodes (which henceforth we refer to as admissions) for England covering the diagnoses made on discharge following admission.

(3) Official death notifications (by date of death) for England and Wales supplied by the Office of National Statistics.

For each age group the weekly GP episode rates, the weekly number of admissions, and of deaths were separately analysed by the decomposition method. By this method any seasonal component of a time series is isolated by first removing the underlying trend, as described by Kendall ${ }^{13}$ and used by Khot and Burn ${ }^{5}$ and by the Lung and Asthma Information Agency ${ }^{14}$ in their studies of seasonal variations in asthma. Here each weekly figure has been expressed as a percentage of the underlying trend value, where the underlying trend has been calculated as a 52 point moving average of the weekly figures. The median of these percentages referable to a given week (over the eight years of available data) is taken as the seasonal index for that week (52 seasonal indices in all). These medians were plotted as three week moving averages to minimise anomalies associated with holiday periods and small numbers of deaths.

\section{Results}

This report is based on material accumulated over eight years. As an example year, there were, in 1994, 15708 GP episodes (WRS population 614000$), 90864$ admissions in England, and 1514 deaths in England and Wales in the respective populations; in children aged $0-14$ years there were only 28 deaths, a number too small for detailed analysis.

GP EPISODES AND HOSPITAL ADMISSIONS

The weekly seasonal indices of GP episodes and of admissions for asthma are presented in fig 1 .

Preschool children (0-4 years)

Six peaks were observed in both data sets, the maximum for GP episodes in early December (week 49) and for admissions in late September (week 38). Both were at a minimum in August (weeks 31-34). The striking temporal congruence of the two measures was matched by congruence in magnitude in all peaks except in September when admissions were $100 \%$ above the average but GP episodes were only $25 \%$ higher.

Children (5-14 years)

Similar peaks to those seen in preschool children were evident in this age group, although there were differences in relative magnitude. Again there was extraordinary congruity between the two series.

Young adults (15-44 years)

GP episodes peaked at 30\% above average at the end of June (week 26) and in late Septem-
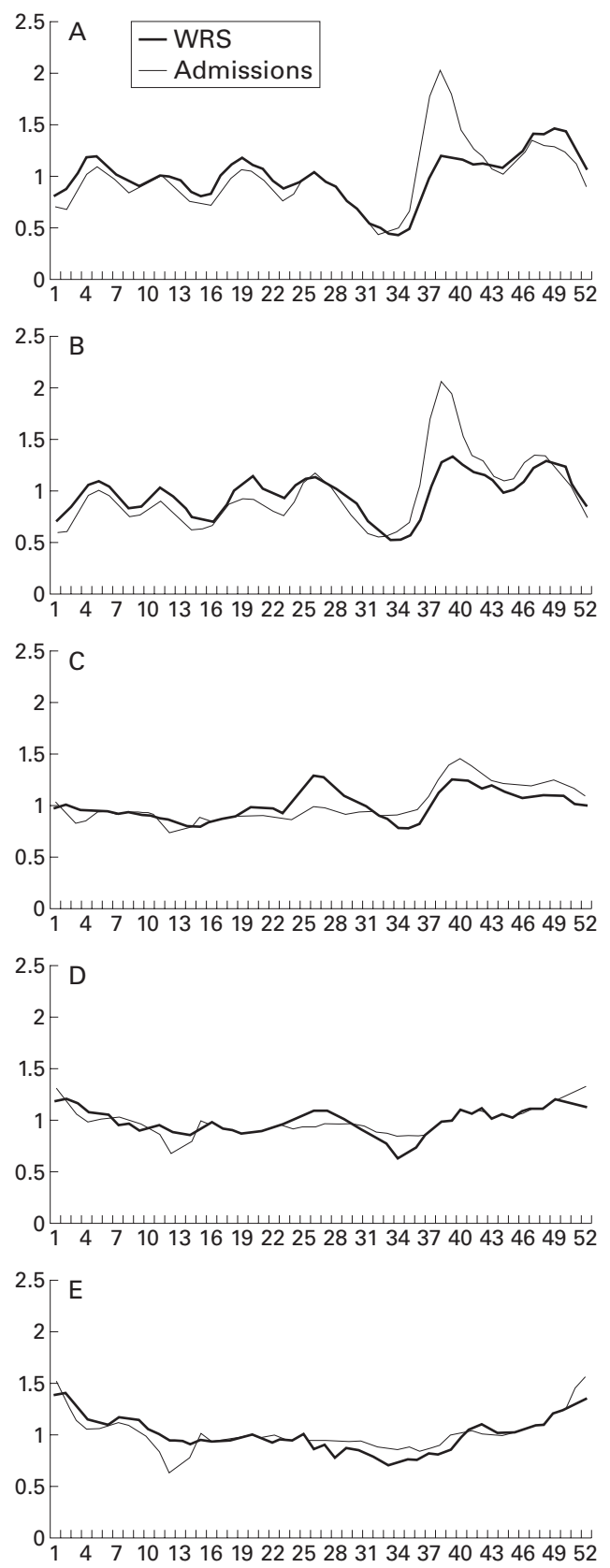

Figure 1 Weekly seasonal indices of general practice episodes and hospital admissions by age group plotted as episodes and hospital admissions by age group plotted as
three week moving averages. (A) 0-4 years; (B) 5-14 years; (C) 15-44 years; (D) 45-64 years; (E) 65 years and over.

ber (week 38) but a peak in hospital admissions only occurred in September.

Middle age (45-64 years)

The midsummer peak in GP episodes (week 26) seen in the younger age groups was also evident in this age group. Admissions were stable during the summer weeks but were higher in mid winter. (A deep trough is evident at week 11 which may be artefactual, see Discussion.)

Elderly (65+ years)

GP episodes and admissions to hospital followed fairly similar patterns with higher values in winter than in summer. 

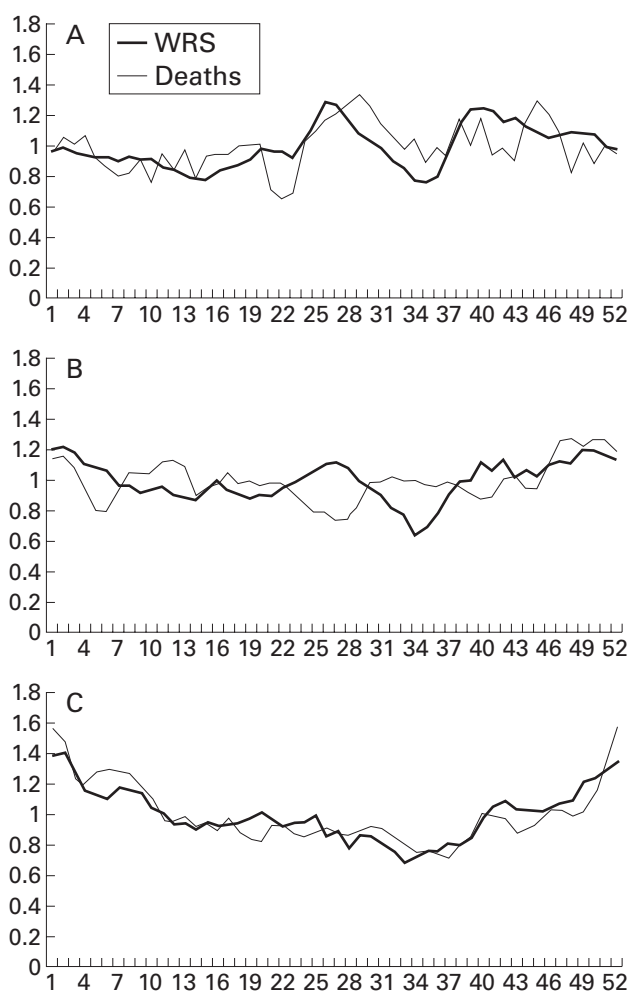

Figure 2 Weekly seasonal indices of general practice episodes and deaths by adult age groups plotted as three week moving averages. (A) 15-44 years; (B) 45-64 years; (C) 65 years and over.

GP EPISODES AND DEATHS

The variation in GP episode rates and deaths from asthma are shown in fig 2 (adults only). In the 15-44 age group there was a substantial peak in mortality in mid summer which lagged two weeks behind the peak in GP episodes. Comparisons between GP episodes and mortality are limited by the small numbers of deaths in any one week (average 3.3, range $0-10$ ), although the figures presented here have been consolidated over eight years. In the 45-64 age group the contrasting findings in June and early July (weeks 22-28; high episodes, low deaths) and mid July to end of September (weeks 29-38; low episodes, average deaths) are the most striking features. Deaths were highest in the last four weeks of the year. In those aged 65 years and over there was a congruity in the data sets throughout the year with sizeable peaks in GP episodes and mortality at the end of the year.

TRENDS WITH INCREASING AGE

Trends are self-evident from consideration of the figures presented above, but we draw particular attention to the following:

GP episodes

In childhood a six peak pattern is clearly evident which, in adults aged 15-44 years, is replaced by two peaks (June/July and September/October) and in those aged over 65 years by a single mid winter peak. In preschool children the maximum values occur in the last four weeks of the year. The May (weeks 18-20) peak in this age group gradually recedes and is lost in adults whereas the mid summer and September peaks increase in prominence in the 5-14 and 15-44 year age groups.

Admissions

The particularly strong peak in September in children aged $0-4$ and $5-14$ years is also evident in the 15-44 year age group but declines thereafter. The spring and mid summer peaks present in children do not persist into adulthood.

\section{Deaths}

The striking feature is the contrast in summer mortality-a peak of deaths in the 15-44 year age group and a trough in those aged 45-64 years. At this time, GP episodes were higher in both age groups but hospital admissions showed little change from average.

\section{Discussion}

This study identifies:

(1) age related variation in the seasonal pattern of asthma presenting in general practice;

(2) inconsistencies in the seasonal relationships between GP episodes of asthma and admissions;

(3) changing relationships between GP episodes, admissions to hospital, and deaths as age increases.

Data collected in the Weekly Returns Service are based on general practice recognition of new episodes. The congruence of temporal variations of these data with those in hospital admissions was striking, especially in children. This suggests that what doctors label as a new episode of asthma is the same whether they work in the community or in hospital. The numbers of episodes presenting to GPs were at their lowest in August. There were very similar reductions in hospital admissions at the same time which were particularly noticeable in school children who were most likely to be on holiday. Thus, it is unlikely that there was a significant bypassing of general practice with direct access to hospital at this time of year. Some anomalies in hospital admission data between weeks 9 and 13 were observed for adults which we suspect must be due to deficiencies in recording of hospital based data towards the end of the financial year (for accountancy purposes, the financial year ends in week 13). We can offer no alternative explanation for the reduction observed at this time.

In young adults (15-44 years) deaths peaked around week 28 in the middle of the pollen season when asthma is unlikely to be a complication of a respiratory infection. This finding is consistent with that reported by Campbell et al for the period 1983-95. ${ }^{7}$ It is surprising that hospital admissions were not increased at this time. Burr et $a l^{15}$ have shown that deaths from asthma in patients under 65 years of age (occurring in Wales between September 1994 and December 1996) generally occurred outside hospital. Weiss also observed an increase in summertime deaths from asthma in the USA which often occurred outside hospital. ${ }^{16} \mathrm{He}$ also noted similarities between the USA and 
the UK in the seasonal patterns of hospital admissions and deaths. Roux and colleagues examined 21 children who presented with acute asthma at a consistent season and compared them with randomly presenting controls. ${ }^{17}$ The consistent group formed a distinct subpopulation with severe asthma (some with a family history of fatal asthma) who were less likely to outgrow asthma in childhood. Robertson and colleagues reporting from Australia studied 163 deaths from asthma, 153 of which occurred outside hospital; 39\% had no previous hospital admission for asthma and just under half were taking inhaled prophylactic medication. ${ }^{18}$ Severe and fatal attacks of asthma can strike in subjects with comparatively minor asthma as well as in those with more severe forms. When deaths are maximal, the illness is either most severe or the acute attacks of asthma are particularly insidious and aggressive.

The adult mortality data (all age groups) do not show peaks in August (weeks 32-35), even though mortality is high relative to GP episodes. However, we draw attention to the limitations of compressing data into four week periods for statistical examination. Consistent peaks occurring over a short period such as four weeks can readily be lost in time series analysis which may divide the peak into separate periods. We also stress the importance of presenting asthma data for children separately from adults as the seasonal patterns are so different.

In all age groups and in all three data sets (GP episodes, admissions, and deaths) we have demonstrated trough values around week 34 (mid August); schools reopen in early September around week 36/37. Whatever exacerbates asthma after week 34 establishes itself before children go back to school. The consistent relationship between episodes and admissions in children is disrupted in magnitude (though not in timing) in the early autumn with a dramatic peak of admissions. A peak of admissions in this season has been observed by others ${ }^{9}{ }^{10}$; the explanation for this peak and its differential impact on GP episodes and hospital admissions merits further investigation.

Silverman has suggested that the name asthma should be changed. ${ }^{19}$ We are used to the concept of clinically indistinguishable respiratory illnesses bearing names that relate to the causative organism. Are the characteristics of spring, autumn and winter asthma different? The precipitating factors are likely to be different and we have found differences in the patterns of health care utilisation in relation to age and risk of admission and of death. The severity of asthma in an individual may be localised to a particular time of the year. It is not known whether an asthmatic child retains the same seasonal pattern into adulthood. We emphasise the need to research the natural history of asthma sufferers to identify seasonal clusters at different periods of their life. The links between virus aggravated wheeze, asthmatic illness in childhood, and intrinsic asthma are tenuous but may have serious implications given the large increase in the prevalence of childhood asthma over the last 20 years. ${ }^{20}$

The seasonal patterns described in this report are useful for patient management, especially to alert individual asthmatic patients to periods of increased risk. We have shown clear differences by age. Our findings suggest that an accurate and carefully recorded seasonal history of when asthma sufferers experience attacks would lead to improvements in patient management, in particular to much more rational use of prophylactic medication and consequent economies in prescribing. The authors are grateful to the recording practices for their per-
severance with continuous morbidity surveillance. They also acknowledge the Department of Health who fund the Weekly Returns Service and have given permission to publish.

1 Sears MR. Epidemiology of childhood asthma. Lancet 1997; 350: $1015-20$

2 McCormick A, Fleming D, Charlton J. Morbidity statistics from general practice. 4th national study 1991-2. Series MB5, No.3. A study carried out by the Royal College of General Practitioners, the Office of Population Censuses and Surveys, and the Department of Health. London: HMSO, 1995.

3 Department of Health. Hospital episode statistics, Volume 1: Finished consultant episodes by diagnosis, operation and specialty. England: financial year 91-92, 92-93. Government Statistical Service, 1994

4 Office of Population Censuses and Surveys. Mortality statistics (cause) England and Wales. Series DH2, No 20. London: HMSO, 1995.

5 Khot A, Burn R. Seasonal variation and time trends of deaths from asthma in England and Wales 1960-82. BMF 1984; 289: 233-4,

6 Campbell MJ, Cogman GR, Holgate ST, et al. Age specific trends in asthma mortality in England and Wales, 1983-95: results of an observational study. BMF 1997; 314: 1439-41.

7 Campbell MJ, Cogman GR, Holgate ST. Trends in asthma mortality. BMF 1997; 315: 1012 .

8 Marks G, Burney P. Diseases of the respiratory system. The health of adult Britain, 1841-1994. Volume 2. London: HMSO, 1997:93-113.

9 Khot A, Burn R, Evans N, et al. Seasonal variation and time rends in childhood asthma in England and Wales 1975-81. BMF 1984; 289: 235-7.

10 Storr J, Lenney W. School holidays and admissions with asthma. Arch Dis Child 1989; 64: 103-7.

11 Fleming DM, Norbury CA, Crombie DL. Annual and seasonal variation in the incidence of common diseases. Occasional paper 53. London: Royal College of General Practitioners, 1991.

12 Fleming DM, Sunderland R, Cross KW, et al. Declining incidence of episodes of asthma: a study of trends in new episodes presenting to general practitioners in the period 1989-98. Thorax 2000;55:657-61.

13 Kendall M. Time series. 2nd ed. London: Griffin, 1976: 55-67.

14 Lung and Asthma Information Agency. Seasonal variations in asthma. Factsheet 9394. London: St George's Hospital Medical School, 1993

15 Burr ML, Davies BH, Hoare A, et al. A confidential inquiry into asthma deaths in Wales. Thorax 1999;54:985-9.

6 Weiss KB. Seasonal trends in US asthma hospitalisation and mortality. FAMA 1990; 263: 2323-8.

17 Roux P, Smit M, Weinberg EG. Seasonal and recurrent intensive care unit admissions for acute severe asthma in children. S Afr Med f 1993; 83: 177-9.

18 Robertson CF, Rubinfeld AR, Bowes G. Deaths from asthma in Victoria: a 12-month survey. Med F Aust 1990; 152: $511-7$.

19 Silverman $M$, Wilson N. Asthma: time for a change of name? Arch Dis Child 1977; 77:62-5.

20 Phelan PD. Asthma in children: epidemiology. BMF 1994:308:1584-5. 